
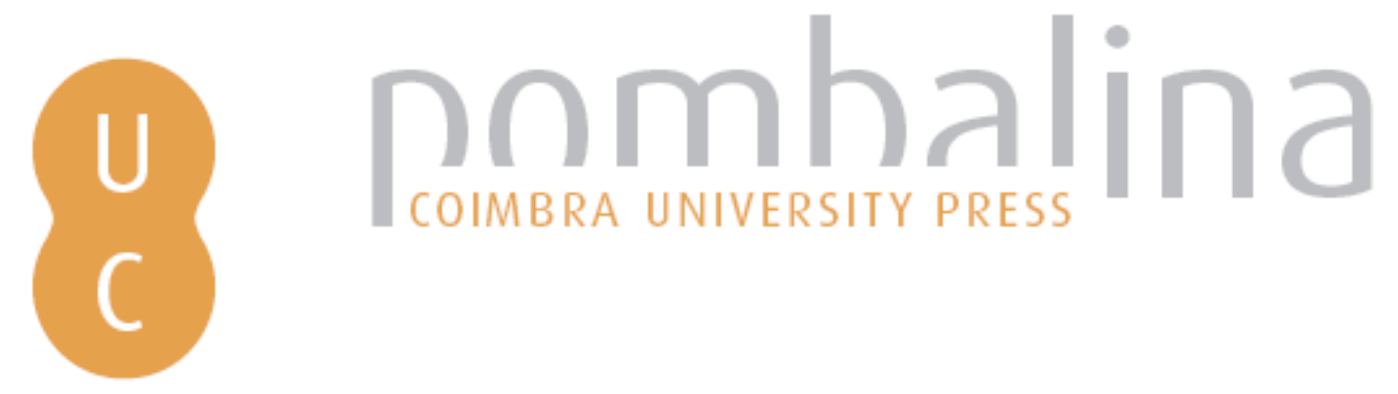

\title{
Desafíos para la implementación clínica de productos de ingeniería de tejidos en latinoamérica
}

\author{
Autor(es): $\quad$ Fontanilla, Marta R.; Suesca, Edward; Casadiegos, Sergio \\ Publicado por: Imprensa da Universidade de Coimbra \\ URL \\ persistente: URI:http://hdl.handle.net/10316.2/36929 \\ DOI: $\quad$ DOI:http://dx.doi.org/10.14195/978-989-26-0881-5_18 \\ Accessed : $\quad$ 26-Apr-2023 12:11:23
}

A navegação consulta e descarregamento dos títulos inseridos nas Bibliotecas Digitais UC Digitalis, UC Pombalina e UC Impactum, pressupõem a aceitação plena e sem reservas dos Termos e Condições de Uso destas Bibliotecas Digitais, disponíveis em https://digitalis.uc.pt/pt-pt/termos.

Conforme exposto nos referidos Termos e Condições de Uso, o descarregamento de títulos de acesso restrito requer uma licença válida de autorização devendo o utilizador aceder ao(s) documento(s) a partir de um endereço de IP da instituição detentora da supramencionada licença.

Ao utilizador é apenas permitido o descarregamento para uso pessoal, pelo que o emprego do(s) título(s) descarregado(s) para outro fim, designadamente comercial, carece de autorização do respetivo autor ou editor da obra.

Na medida em que todas as obras da UC Digitalis se encontram protegidas pelo Código do Direito de Autor e Direitos Conexos e demais legislação aplicável, toda a cópia, parcial ou total, deste documento, nos casos em que é legalmente admitida, deverá conter ou fazer-se acompanhar por este aviso.

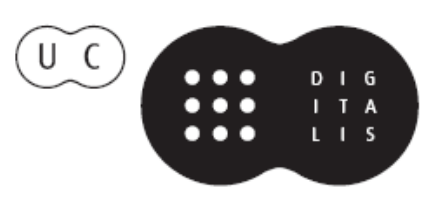


IOMATERIAIS APLICADOS AO DESENVOLVIMENTO DE SISTEMAS TERAPÊUTICOS AVANÇADOS

IOMATERIALES APLICADOS AL DISEÑO DE SISTEMAS TERAPÉUTICOS AVANZADOS

Hermínio C. de Sousa Mara E. M. Braga Alejandro Sosnik (editores) 


\section{CAPÍTULO 18. DESAFÍOS PARA LA IMPLEMENTACIÓN CLÍNICA DE PRODUCTOS DE INGENIERÍA DE TEJIDOS Y MEDICINA REGENERATIVA EN LATINOAMÉRICA}

Marta R. Fontanilla, Edward Suesca, Sergio Casadiegos Grupo de Trabajo en Ingeniería de Tejidos, Laboratorio 318, Departamento de Farmacia, Universidad Nacional de Colombia. Avda Carrera 30 \# 45-03, Bogotá, Colombia.

\section{Resumen:}

La ingeniería de tejidos y la medicina regenerativa (ITMR) usan soportes, células y factores de crecimiento, solos o combinados, para reparar o regenerar tejidos lesionados o perdidos. Los principales avances en ITMR se han hecho en los países desarrollados, a pesar de que los problemas de salud que solucionan estas tecnologías prevalecen en países en desarrollo como los nuestros. Por ésta razón, es importante conocer que ocurre en investigación, desarrollo e implementación de terapias basadas en productos ITMR en la región. En éste capítulo se revisa la investigación, transferencia y producción de medicamentos ITMR (incluyendo información sobre los bancos de sangre de cordón), en Latinoamérica. La búsqueda llevada a cabo, demuestra la necesidad de formar más personal en el área y aumentar el apoyo financiero a la investigación y desarrollo, ya que aunque las tecnologías ITMR que son costosas pueden llegar a impactar favorablemente a los sistemas de salud de la región.

Palabras clave: Ingeniería de tejidos en Latinoamérica; implementación de terapias regenerativas; ingeniería de tejidos; medicina regenerativa. 


\begin{abstract}
:
Tissue engineering and regenerative medicine (TERM) approaches use scaffolds, cells and growth factors, alone or combined, to promote repair or regeneration of tissue that has been injured or lost. Major advances in TERM research and translation have been made in developed countries although the health problems that TERM-based therapies solve prevail in developing regions like ours. Therefore, identifying the challenges faced by Latin American scientists working in the field becomes a priority. The aim of this chapter was to survey research groups, clinical translation initiatives, TERM products manufactured locally and cord blood banks found in the region. The survey carried out indicates that TERM development in the region demands training of human resources and the increase of financial support. This effort must be made despite their high cost, because implementation of TERM technologies can have a good impact in the health systems of the Latin American countries.
\end{abstract}

Keywords: Tissue engineering in Latin America; implementation of regenerative therapies; tissue engineering; regenerative medicine. 


\subsection{Introducción}

La ingeniería de tejidos es un campo multidisciplinario, cuyo objetivo es desarrollar tejidos y órganos artificiales que al injertarse estimulen la regeneración morfológica y funcional de los tejidos y órganos que reemplazan [1, 2]. Al congregar diferentes profesiones, crea el espacio necesario para encontrar soluciones a los desafíos que se presentan cuando se quiere imitar a la naturaleza; sin embargo, por ahora sus alcances están limitados por la capacidad que sus productos tienen de estimular la regeneración del cuerpo y por su baja implementación clínica.

Estados Unidos fue el país que desarrolló y aprobó los primeros sustitutos de tejidos para el tratamiento de lesiones de piel, hueso y cartílago en humanos [3, 4]; igualmente, inició la investigación y desarrollo de sustitutos artificiales de otros tejidos y órganos del cuerpo [5, 6]. Los beneficios terapéuticos de su aplicación, despertaron el interés mundial en el tema; por lo cual, se crearon centros académicos y grupos de investigación, asociados o no a universidades, con el fin de investigar e innovar en ésta área. La aplicación clínica de los resultados investigativos, condujeron a la formación de compañías que después de escalar la producción de sustitutos (e.g: Integra ${ }^{\circledR}$, Apligraf $₫$ y Carticel®), lograron su comercialización [7, 8]. Actualmente, países considerados emergentes cuyas economías están dentro de las de mayor crecimiento, como India y China [9], también investigan y desarrollan productos de ingeniería de tejidos constituyéndose en centros importantes de innovación.

Más de 30 años de investigación y algunos de aplicación de este tipo de productos, han mostrado su efecto en favorecer el poder regenerador del cuerpo [10]. Lo anterior sumado al descubrimiento de las bondades de las células madre y de los factores secretados por ellas y otras células, para promover la regeneración de órganos y tejidos, llevó a que la ingeniería de tejidos y la terapia celular confluyeran en un campo de gran potencial hoy conocido como Medicina Regenerativa $[11,12]$. Los productos de ingeniería de tejidos y medicina regenerativa (ITMR), varían en complejidad dependiendo del tipo de lesión y del órgano o tejido en que se van a aplicar. Por ejemplo, colocar soportes 
tridimensionales porosos de biomateriales biodegradables en pérdidas de continuidad de piel, mucosa o hueso promueve su regeneración; mientras que un órgano como la vejiga no puede ser reemplazado únicamente por el soporte, ya que necesita de componentes celulares que in situ estimulen su recambio y regeneración [5, 6, 13, 14]. Como consecuencia de sus diferencias en complejidad, la gama de productos ITMR comercializados o en desarrollo es muy variada y amplia.

Los problemas de salud tratados con medicina regenerativa, incluida la ingeniería de tejidos, prevalecen en los países emergentes; sin embargo, la mayoría de la investigación e innovación en este campo se ha llevado a cabo en los países desarrollados [15]. De ahí, la importancia de identificar los factores que limitan su aplicación en el tratamiento de los problemas de salud que afectan a países en desarrollo, como los nuestros. Con tal fin, este capítulo habla sobre la naturaleza de los productos ITMR, presenta el estado del campo en Latinoamérica, discute algunos de los desafíos que se enfrentan en la implementación de la ingeniería de tejidos y la medicina regenerativa en esta zona y señala algunas aproximaciones a seguir para conseguir que los países latinoamericanos participen activamente en sus desarrollos y beneficios.

\subsection{Naturaleza y regulación de los productos de ingeniería de tejidos}

Se han desarrollado una gran diversidad de productos de ingeniería de tejidos constituidos por células, soportes, factores de crecimiento, solos o combinados, los cuales fundamentan las aproximaciones terapéuticas de la medicina regenerativa. Su complejidad varía dependiendo de la función que desempeñan cuando se colocan en el organismo, su composición y la tecnología utilizada para su manufactura. Por ejemplo, un soporte tridimensional elaborado con un material biodegradable en el que no se han sembrado y cultivado células, es más simple que aquel en el que se han sembrado; por ende, los requerimientos de las agencias regulatorias de medicamentos para aprobar su comercialización son diferentes. 
Debido a que muchos de estos productos están constituidos por tejidos tratados y células vivas o sus derivados, los productos ITMR conforman un grupo especial dentro de los medicamentos biológicos y los dispositivos médicos. Su común denominador es que son bioactivos y no son producidos con los métodos químicos usados en la manufactura de los medicamentos convencionales (los cuales integran un principio activo o fármaco sintético, semi-sintético o natural en una forma farmacéutica). Su bioactividad depende del tipo de producto y se manifiesta en que señalizan a las células del sitio implantado, para que desencadenen las respuestas biológicas que activan la capacidad de regeneración del cuerpo [16, 17]. Es importante notar que la regulación de otros medicamentos biológicos como las proteínas recombinantes, ha sido complicada y muy controversial; por lo tanto, es de esperar que los productos ITMR de mayor complejidad que estas últimas, tengan que recorrer un camino sinuoso antes de que existan regulaciones armonizadas que garanticen que en los diferentes países se produzcan con características de eficacia y seguridad similares.

Algunos de los productos ITMR han sido aprobados para comercialización como dispositivos médicos. Sin embargo, la Farmacopea de los Estados Unidos (USP) incluye a los tejidos formados por soportes y células en el grupo de "Productos derivados de células y tejidos" y solo considera como dispositivos médicos a las membranas o soportes acelulares elaboradas con biomateriales sintéticos o naturales; exceptuando, los soportes obtenidos a partir de tejidos humanos por diferentes tratamientos [18]. La Administración de Medicamentos y Alimentos (FDA) de los Estados Unidos incluye a los productos de ingeniería de tejidos y medicina regenerativa dentro de los medicamentos biológicos, clasificándolos en: i) Células humanas, tejidos y productos basados en células y tejidos [Human cells, tissues, and cellular and tissue-based products (HCT/P)]; ii) Combinación de HCT/P y dispositivos; iii) Productos de terapia celular somática y de terapia génica. La FDA no considera como productos HCT/P a los tejidos humanos o células para uso autólogo que han sido removidos y posteriormente implantados durante el mismo procedimiento quirúrgico, a las células reproductivas o a los tejidos para transferir a un compañero sexual íntimo, a las células o tejidos donados como parte de un programa de tec- 
nología de reproducción asistida, a los órganos donados para trasplante, a la sangre o componentes de ella (Células rojas, células blancas, plaquetas y plasma para transfusión), excepto células progenitoras hematopoyéticas, y a los tejidos o células humanas que se utilicen en investigaciones científicas no-clínicas o con propósitos educativos [19].

La Agencia Europea de Medicamentos (EMA), afirma que "los progresos en biotecnología celular y molecular han llevado al desarrollo de terapias avanzadas, como la terapia génica, terapia celular somática e ingeniería de tejidos”. Reconoce que este tipo de productos va más allá del ámbito farmacéutico y del ámbito de los productos biológicos y los dispositivos médicos; en consecuencia, a los productos empleados en este tipo de terapias los agrupa y clasifica como medicamentos de terapias avanzadas [20].

Inicialmente los productos ITMR fueron aprobados por la FDA como dispositivos médicos y actualmente aquellos de baja complejidad como los soportes elaborados con biomateriales degradables aun se clasifican en esta categoría [21], por lo tanto en su regulación se han tenido en cuenta los principios de clasificación de dispositivos médicos establecidos por la Fuerza Especial para la Armonización Global [Global Harmonization Task Force (GHTF)], un grupo voluntario constituido por representantes de agencias regulatorias [22]. Hasta el momento en Colombia el Instituto Nacional de Vigilancia de Medicamentos y Alimentos (INVIMA), solo ha regulado a los productos ITMR que solicitan registro como dispositivos médicos; para esto, incluyó la clasificación propuesta por la GHTF en el decreto 4725 del 2005, elaborado por el Ministerio de la Protección Social. El decreto en mención establece que debido al tiempo que duran en contacto con el cuerpo, el grado de invasión y la relación que existe entre el efecto local y el efecto sistémico, los soportes empleados en ingeniería de tejidos deben ser clasificados como dispositivos médicos clase III. Está en curso la aprobación de un proyecto de decreto sobre medicamentos biológicos y biotecnológicos que incluye a los productos de ingeniería de tejidos y medicina regenerativa [22]. En general se puede considerar que debido a su reciente desarrollo y aplicación, en los países latinoamericanos hasta ahora se está implementado la utilización y reglamentación de los productos ITMR. 


\subsection{Estado actual de la ingeniería de tejidos y la medicina re- generativa}

Las lesiones de cartílago articular y las pérdidas de continuidad de piel y mucosa, son frecuentes en todas las poblaciones del planeta. Sin embargo, los países con ingresos bajos y moderados, que representan el $85 \%$ de la población mundial, enfrentan el $90 \%$ de muertes ocasionadas por lesiones o trauma y poseen el $80 \%$ de las personas que en el mundo sufren algún tipo de discapacidad [23]. En estos países las prácticas saludables son poco comunes, además, la prevención y el acceso a una atención en salud de calidad en los estratos socieconómicos medios y bajos son limitados [24]. En consecuencia, las enfermedades crónicas que resultan en el daño de órganos o en pérdida de tejidos son frecuentes. Por ejemplo, se ha postulado que entre el 2000 y el 2020, el porcentaje de personas con cáncer habrá aumentado el $73 \%$ en los países en desarrollo mientras que en los desarrollados el incremento será solo del $29 \%$ [25, 26]. Además, los conflictos sociales y la violencia incrementan la incidencia de heridas por quemadura, explosiones, minas antipersonales, armas de fuego y armas cortopunzantes [27].

Muchos de los problemas de salud arriba descritos, se tratan con trasplantes de órganos o injertos de tejidos. La escasez de donantes y las complicaciones asociadas con la utilización de tejidos que no son autólogos (homo y xenoinjertos), han impulsado la elaboración in vitro de órganos y tejidos; sin embargo, la complejidad de algunos de estos ha obstaculizado su obtención y su uso clínico. En consecuencia órganos complejos, como las glándulas o el riñón, hasta el momento no han podido ser llevados del laboratorio de investigación a la práctica clínica [15]. A pesar de lo anterior, en el siglo XXI se han tratado con productos ITMR pacientes que presentan lesiones de piel, cartílago, hueso, válvulas cardiacas, vejiga, esófago, zonas infartadas de corazón, córnea y tráquea [28-32]. Los resultados clínicos de estos tratamientos, mejores que los obtenidos con los métodos convencionales, son los que han promovido el crecimiento de la ingeniería de tejidos y de la medicina regenerativa en el mundo y en Latinoamérica. 
Un estudio sobre los beneficios potenciales de la medicina regenerativa en los sistemas de salud de países de ingresos medios y bajos, reportó la existencia de grupos de investigación, generalmente asociados con universidades, que trabajan en el campo. Por el contrario, su éxito en la creación de compañías dedicadas a ofrecer productos y servicios en ingeniería de tejidos evidenció ser muy bajo [33, 34]. Para mostrar el panorama actual de la ingeniería de tejidos y medicina regenerativa en Latinoamérica, hicimos una búsqueda en internet a través del motor de búsqueda Google asociando el nombre de cada país latinoamericano a cada una de las siguientes palabras claves: Ingeniería de tejidos, tejido artificial, medicina regenerativa, terapia celular y células madre. Adicionalmente, se revisaron los eventos académicos en las áreas de ingeniería biomédica, biomateriales, ingeniería de tejidos y medicina regenerativa para identificar a los latinoamericanos participantes y se buscó en la base de datos MedLine la producción bibliográfica de los grupos de investigación identificados. La información encontrada se organizó en tres secciones: Investigación, producción comercial y bancos de sangre de cordón umbilical.

\subsubsection{Investigación en ingeniería de tejidos y medicina regenerativa}

Durante la revisión se encontró que hay grupos vinculados a universidades y centros de investigación que investigan en ITMR. La información del grupo, los temas de investigación y la institución a la que pertenece, fue agrupada por país y es presentada en la Tabla 18.1. En ella se observa que en 10 de los 20 países Latinoamericanos, se realizan investigaciones con el potencial de desarrollar productos ITMR.

\subsubsection{Producción comercial}

Se buscó información sobre empresas netamente latinoamericanas que produjeran y comercializaran productos ITMR. Los datos encontrados (Tabla 18.2), muestran que hay empresas latinoamericanas dedicadas a 
las siguientes actividades: producción de dispositivos médicos utilizando biomateriales biodegradables; aplicación de terapias con plasma rico en plaquetas o células madre mesenquimales derivadas de médula ósea; láminas de queratinocitos (Autólogos y homólogos) y cartílago. Además, laboratorios Craveri en Argentina, aunque no cuenta con productos ITMR en el mercado, fue la primera empresa de la región en incorporar una división de bioingeniería en el año 1995 y ahora tiene líneas de investigación en cartílago, oftalmología y piel; sin embargo, parte de sus desarrollos todavía se encuentran en ensayos clínicos y otros están esperando su aprobación para ser comercializados [29].

Tabla 18.1. Grupos Latinoamericanos de investigación en ITMR.

\begin{tabular}{|c|c|c|}
\hline Grupo & Investigación & Institución \\
\hline \multicolumn{3}{|l|}{ Argentina } \\
\hline $\begin{array}{l}\text { Centro de Investigación en In- } \\
\text { geniería de Tejidos y Terapias } \\
\text { Celulares (CIITTC) }[35,36] .\end{array}$ & $\begin{array}{l}\text { Páncreas, sistema cardiovascular y } \\
\text { nervioso. }\end{array}$ & $\begin{array}{l}\text { Universidad Maimónides, } \\
\text { Buenos Aires. }\end{array}$ \\
\hline $\begin{array}{l}\text { Laboratorio de Ingeniería } \\
\text { Tisular y Medicina Regenera- } \\
\text { tiva [37-39]. }\end{array}$ & $\begin{array}{l}\text { Células madre, ingeniería de teji- } \\
\text { dos de piel. }\end{array}$ & $\begin{array}{l}\text { CUCAIBA (Centro Único } \\
\text { Coordinador de Ablación e } \\
\text { Implante, Buenos Aires) }\end{array}$ \\
\hline $\begin{array}{l}\text { Instituto de Investigaciones } \\
\text { en Ciencia y Tecnología de } \\
\text { Materiales (INTEMA) }[35,40] \text {. }\end{array}$ & $\begin{array}{l}\text { Biomateriales, soportes y nanofi- } \\
\text { bras para ingeniería de tejidos. }\end{array}$ & $\begin{array}{l}\text { Facultad de Ingeniería de } \\
\text { la Universidad Nacional de } \\
\text { Mar del Plata }\end{array}$ \\
\hline \multicolumn{3}{|l|}{ Brasil } \\
\hline $\begin{array}{l}\text { Biomateriais para Implante } \\
\text { em tecido ósseo }[41,42] .\end{array}$ & $\begin{array}{l}\text { Biomateriales e ingeniería de tejido } \\
\text { óseo. }\end{array}$ & $\begin{array}{l}\text { Universidade de São Paulo, } \\
\text { Faculdade de Odontologia } \\
\text { de Ribeirão Preto }\end{array}$ \\
\hline $\begin{array}{l}\text { Reparo e Biocompatibilidade } \\
{[43,44] .}\end{array}$ & $\begin{array}{l}\text { Sistema óseo y biomateriales para } \\
\text { uso odontológico y médico. }\end{array}$ & $\begin{array}{l}\text { Universidade de São Paulo, } \\
\text { Faculdade de Odontologia } \\
\text { de Ribeirão Preto }\end{array}$ \\
\hline $\begin{array}{l}\text { Laboratório de Investigação } \\
\text { Médica do Sistema Músculo } \\
\text { Esquelético }[45,46] .\end{array}$ & $\begin{array}{l}\text { Regeneración del sistema musculo } \\
\text { esquelético. }\end{array}$ & Universidade de São Paulo. \\
\hline $\begin{array}{l}\text { Heart Institute (InCor) [47, } \\
48] .\end{array}$ & $\begin{array}{l}\text { Ingeniería de tejidos del sistema } \\
\text { cardiovascular. }\end{array}$ & $\begin{array}{l}\text { Universidade de São Paulo. } \\
\text { Escuela de Medicina. }\end{array}$ \\
\hline \multicolumn{3}{|l|}{ Chile } \\
\hline $\begin{array}{l}\text { Centro de Investigación en } \\
\text { Criogenia e Ingeniería de Teji- } \\
\text { dos en Chile (Cicrit) }[49,50] . \\
\end{array}$ & Regeneración de piel. & Universidad de Valparaíso. \\
\hline $\begin{array}{l}\text { Biorreactores y Biosensores } \\
{[16,51,52] .}\end{array}$ & $\begin{array}{l}\text { Biorreactores para la producción } \\
\text { de Factores de crecimiento. }\end{array}$ & $\begin{array}{l}\text { Centro de Biotecnología } \\
\text { de la Universidad Técnica } \\
\text { Federico Santa María. }\end{array}$ \\
\hline $\begin{array}{l}\text { Centro de Investigación Bio- } \\
\text { médica [53]. }\end{array}$ & $\begin{array}{l}\text { Tratamiento de úlceras crónicas en } \\
\text { pie diabético. }\end{array}$ & $\begin{array}{l}\text { Universidad Austral de } \\
\text { Chile - Fundación Instituto } \\
\text { Nacional de Heridas. }\end{array}$ \\
\hline Departamento de Polímeros [53]. & Aplicaciones biomédicas de quitosán. & $\begin{array}{l}\text { Universidad de Concepción, } \\
\text { Facultad de Ciencias Químicas. }\end{array}$ \\
\hline
\end{tabular}




\begin{tabular}{|c|c|c|}
\hline Grupo & Investigación & Institución \\
\hline \multicolumn{3}{|l|}{ Colombia } \\
\hline $\begin{array}{l}\text { Grupo de Trabajo en Ingeniería } \\
\text { de Tejidos (GTIT) }[16,54,55] \text {. }\end{array}$ & $\begin{array}{l}\text { Biosensores en ITMR, biorreactores, } \\
\text { soportes para ingeniería de tejidos de } \\
\text { mucosa oral, ocular, cartílago y piel. }\end{array}$ & $\begin{array}{l}\text { Universidad Nacional de } \\
\text { Colombia. }\end{array}$ \\
\hline $\begin{array}{l}\text { Biología de Células Madre } \\
\text { [42]. }\end{array}$ & $\begin{array}{l}\text { Células madres mesenquimales y } \\
\text { su potencial angiogénico. }\end{array}$ & $\begin{array}{l}\text { Universidad Nacional de } \\
\text { Colombia. }\end{array}$ \\
\hline $\begin{array}{l}\text { Grupo de Ingeniería Biomédi- } \\
\text { ca }[56,57] \text {. }\end{array}$ & $\begin{array}{l}\text { Biomecánica, hemosustitutos. Inge- } \\
\text { niería de tejidos y biomateriales }\end{array}$ & Universidad de los Andes \\
\hline $\begin{array}{l}\text { Grupo Ingeniería de tejidos y } \\
\text { terapias celulares }[58,59] \text {. }\end{array}$ & $\begin{array}{l}\text { Producción de sustitutos biológicos } \\
\text { Terapias celulares }\end{array}$ & Universidad de Antioquia. \\
\hline $\begin{array}{l}\text { Grupo de Investigación en } \\
\text { Biomateriales - BIOMAT [60]. }\end{array}$ & $\begin{array}{l}\text { Biomateriales, soportes para } \\
\text { ingeniería de tejidos, materiales } \\
\text { biocerámicos. }\end{array}$ & Universidad de Antioquia. \\
\hline Terapia Regenerativa [61, 62]. & $\begin{array}{l}\text { Concentrados de plaquetas en } \\
\text { patología músculo-esquelética, } \\
\text { modelos animales de enfermedad } \\
\text { músculo-esquelética. }\end{array}$ & Universidad de Caldas. \\
\hline $\begin{array}{l}\text { Grupo de Investigación en } \\
\text { Ingeniería Biomédica EIA-CES } \\
\text { (GIBEC) }[48,63] \text {. }\end{array}$ & $\begin{array}{l}\text { Biotecnología en salud y biomate- } \\
\text { riales. }\end{array}$ & Universidad CES. \\
\hline \multicolumn{3}{|l|}{ Costa Rica } \\
\hline $\begin{array}{l}\text { Laboratorio de Ingeniería de } \\
\text { Tejidos [64]. }\end{array}$ & Producción de piel artificial. & $\begin{array}{l}\text { Centro de Investigación en } \\
\text { Biotecnología del Instituto } \\
\text { Tecnológico Costa Rica } \\
\text { (TEC) }\end{array}$ \\
\hline \multicolumn{3}{|l|}{ Cuba } \\
\hline Centro de Biomateriales $[15,65]$. & Biomateriales & Universidad de la Habana \\
\hline $\begin{array}{l}\text { Centro de Ingeniería Genética } \\
\text { y Biotecnología [66]. }\end{array}$ & $\begin{array}{l}\text { Medicina regenerativa mediante } \\
\text { factores de crecimiento recombi- } \\
\text { nantes. }\end{array}$ & $\begin{array}{l}\text { Centro de Ingeniería Gené- } \\
\text { tica y Biotecnología }\end{array}$ \\
\hline \multicolumn{3}{|l|}{ Ecuador } \\
\hline $\begin{array}{l}\text { Laboratorio Acelerador de } \\
\text { Electrones [67]. }\end{array}$ & $\begin{array}{l}\text { Aplicaciones de un acelerador de } \\
\text { electrones en la obtención de piel. }\end{array}$ & $\begin{array}{l}\text { Escuela Politécnica Nacio- } \\
\text { nal. }\end{array}$ \\
\hline \multicolumn{3}{|l|}{ México } \\
\hline $\begin{array}{l}\text { Unidad de Ingeniería de } \\
\text { Tejidos, Terapia Celular y Me- } \\
\text { dicina Regenerativa }[18,68] .\end{array}$ & Regeneración ósea. & $\begin{array}{l}\text { Instituto Nacional de Reha- } \\
\text { bilitación. }\end{array}$ \\
\hline $\begin{array}{l}\text { Grupo de Investigación en } \\
\text { Ingeniería Tisular [69]. }\end{array}$ & $\begin{array}{l}\text { Ingeniería de tejidos de piel y } \\
\text { hueso }\end{array}$ & $\begin{array}{l}\text { Universidad Autónoma de } \\
\text { San Luis Potosí. }\end{array}$ \\
\hline $\begin{array}{l}\text { Unidad Guaymas en Asegura- } \\
\text { miento de Calidad y Apro- } \\
\text { vechamiento Sustentable de } \\
\text { Recursos Naturales }[70,71] \text {. }\end{array}$ & $\begin{array}{l}\text { Ingeniería de tejidos de piel, apli- } \\
\text { caciones biomédicas de quitosán. }\end{array}$ & $\begin{array}{l}\text { Centro de Investigación en } \\
\text { Alimentación y Desarrollo. }\end{array}$ \\
\hline $\begin{array}{l}\text { Laboratorio de Inmunoterapia } \\
\text { e ingeniería de Tejidos [72]. }\end{array}$ & Ingeniería de tejidos de piel y hueso & $\begin{array}{l}\text { Universidad Autónoma de } \\
\text { México. }\end{array}$ \\
\hline \multicolumn{3}{|l|}{ Uruguay } \\
\hline Facultad de Química [73]. & $\begin{array}{l}\text { Ingeniería de tejidos de piel, so- } \\
\text { portes a base de colágeno }\end{array}$ & Universidad de la República \\
\hline \multicolumn{3}{|l|}{ Venezuela } \\
\hline $\begin{array}{l}\text { Departamento de Biología } \\
\text { Celular [74]. }\end{array}$ & $\begin{array}{l}\text { Ingeniería de tejidos de cartílago } \\
\text { y piel }\end{array}$ & Universidad Simón Bolívar, \\
\hline $\begin{array}{l}\text { Laboratorio de Bioingeniería } \\
\text { de Tejidos }[75,76] .\end{array}$ & $\begin{array}{l}\text { Regeneración ósea, ingeniería de } \\
\text { tejidos de piel, regeneración ner- } \\
\text { vios periféricos }\end{array}$ & Universidad Simón Bolívar. \\
\hline
\end{tabular}


Algunas de las compañías incluidas en la Tabla 18.2, tienen origen en grupos de investigación vinculados a universidades, públicas o privadas, o licencian tecnologías desarrolladas en ellas. Ejemplo de esto son Biomaster, una spin-off de la Universidad de los Andes en Bogotá, Colombia, y Recalcine, compañía chilena que licenció productos desarrollados en la Universidad de Concepción, Chile [77]. La mayoría de estos productos están indicados para el tratamiento de lesiones de piel y de cartílago.

\subsubsection{Bancos de sangre de cordón umbilical}

En la última década ha aumentado el interés por la sangre de cordón umbilical como fuente de células madre hematopoyéticas y mesenquimales, lo cual se refleja en la implementación a nivel mundial de bancos privados y públicos para su criopreservación. En muchos países los bancos privados de sangre de cordón umbilical han surgido debido a la oportunidad de negocio, creada por la promesa de curar con células madre enfermedades graves como leucemia, diabetes, autoinmunidades y otros padecimientos o lesiones. Hasta el momento estas células solo han sido aprobadas para el trasplante de médula ósea, en el tratamiento de desórdenes hematológicos y del sistema inmune; sin embargo, su eficacia y seguridad en el tratamiento de otras patologías aún no ha sido demostrada [78]. La Sociedad Argentina de Hematología ha manifestado "que la utilidad demostrada de las células progenitoras hematopoyéticas de cordón umbilical es el trasplante alogénico como reconstituyente hematopoyético y que no existen evidencias concluyentes sobre la utilización de estas células para la reconstitución de otros tejidos". Además "la Sociedad promueve y apoya el sistema voluntario, altruista y solidario sobre el que se basa el programa argentino de donación de órganos y tejidos”.

El primer banco de sangre de cordón se estableció en el año 1993 en el centro de sangre de Nueva York, iniciativa pública que hasta el momento se mantiene como el banco de sangre de cordón más grande del mundo [79]. Los bancos encontrados en Latinoamérica, localizados en nueve países, son incluidos en la Tabla 18.3. El primer banco latinoame- 
ricano, fue creado en la Argentina en 1996 en el Hospital de Pediatría Garrahan como un programa relacionado (familiar) de banco de sangre de cordón; en la misma institución, más adelante en el 2005 surgió el programa no relacionado (público) [80]. En Argentina, INCUCAI (Instituto Nacional Centro Único Coordinador, Ablación e Implante) perteneciente al Ministerio de Salud de la Nación, tiene un programa de trasplante de progenitores hematopoyéticos, que incluye al banco de sangre de cordón del Hospital de Pediatría Garrahan, así como otros centro de colecta, criopreservación y trasplante de médula ósea del país.

En el año 2001, México vio surgir su primer banco de sangre de cordón umbilical; esta entidad privada, en la actualidad cuenta con filiales en 28 oficinas en las principales ciudades de México, Colombia, Brasil y Estados Unidos [81]. Fue también en México donde se creó el primer banco latinoamericano público (CordMX), el cual se fundó en la ciudad de México en el 2003, como parte del Centro Nacional de la Transfusión Sanguínea, dependiente de la Secretaría de Salud [82].

Los únicos países en Latinoamérica, además de México y Argentina, que cuentan con bancos públicos son Brasil y Colombia. Sin embargo, ningún banco de cordón latinoamericano público o privado, está en la lista de bancos acreditados por la Fundación para la Acreditación de Terapia Celular (FACT), creada por NETCORD con el propósito de establecer estándares internacionales de calidad y seguridad. NETCORD es la red encargada de mantener el registro internacional de bancos de sangre de cordón umbilical y de las unidades de sangre de cordón que ellos almacenan. En el mundo, FACT es la única entidad con autoridad para acreditar la colecta, procesamiento, evaluación, almacenamiento, selección y aprobación de unidades de cordón umbilical. Se debe resaltar que hasta el momento, FACT solo ha acreditado 47 bancos de sangre de 19 países: Australia, Austria, Bélgica, Canadá, Francia, Alemania, Grecia, Hong Kong, Israel, Italia, Holanda, San Marino, Singapur, España, Suiza, Suecia, Taiwán, Reino Unido y Estados Unidos [85]. Aunque los bancos de cordón brasileños no están acreditados por FACT, este es el único país latinoamericano que posee un programa de terapia celular basado en células progenitoras hematopoyéticas de médula ósea, u obtenidas por aféresis, acreditado por esta entidad [86]. 
Tabla 18.2. Compañías que producen u ofrecen productos ITMR en Latinoamérica.

\begin{tabular}{|c|c|c|}
\hline Empresa & Producto/ Descripción & Uso \\
\hline \multicolumn{3}{|l|}{ Argentina } \\
\hline $\begin{array}{l}\text { Laboratorios } \\
\text { Celina }[83]\end{array}$ & 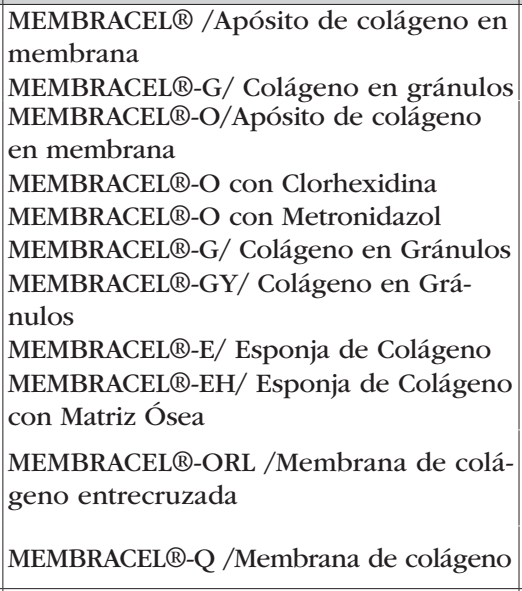 & $\begin{array}{l}\text { Cirugía maxilofacial, implantología y } \\
\text { enfermedad periodantal. Cobertura de } \\
\text { cualquier relleno en cavidad de tejido } \\
\text { óseo, cobertura de implante rodeado } \\
\text { por coágulo sanguíneo, cobertura de } \\
\text { alveolo post-extracción. }\end{array}$ \\
\hline $\begin{array}{l}\text { Hyaltec } \\
\text { Argentina } \\
\text { S.A [84] }\end{array}$ & $\begin{array}{l}\text { Jaloskin/ Film transparente de HYAFF } \\
\text { (ester bencílico de ácido hialurónico) } \\
\text { Hyalogran/ Microgránulos de alginato } \\
\text { de sodio y HYAFF } \\
\text { Hyalofill-F/ Soporte de HYAFF } \\
\text { Hyalomatrix-PA/ Soporte de HYAFF } \\
\text { cubierto por una capa de silicona } \\
\text { Hyalofast/ Soporte de HYAFF y un } \\
\text { importante componente de cartílago } \\
\text { humano } \\
\text { Hyalonect/ malla de HYAFF } \\
\text { Hyaloglide/ gel de ácido hilarónico } \\
\text { Hyalograft 3D/ soporte de HYAFF con } \\
\text { fibroblastos autólogos } \\
\text { Laseskin/ / soporte de HYAFF con mi- } \\
\text { croperforaciones efectuadas con sistema } \\
\text { láser y queratinocitos autólogos }\end{array}$ & $\begin{array}{l}\text { Úlceras, quemaduras, heridas quirúrgi- } \\
\text { cas y por trauma } \\
\text { Úlceras y heridas profundas con abun- } \\
\text { dante exudado } \\
\text { Úlceras y preparación del lecho para el } \\
\text { implante de injertos de piel } \\
\text { Quemaduras profundas o pérdidas } \\
\text { cutáneas de espesor total } \\
\text { Atrapamiento de células madre mesen- } \\
\text { quimales para la reparación de lesiones } \\
\text { condrales y osteocondrales } \\
\text { Rápida estabilización del material de } \\
\text { hueso injertado } \\
\text { Barrera contra la formación de adhe- } \\
\text { rencias post-operatorias de tendón y } \\
\text { nervios } \\
\text { Úlceras, quemaduras, heridas quirúrgi- } \\
\text { cas y por trauma, cirugía plástica } \\
\text { Reemplazar autoinjerto en úlceras, que- } \\
\text { maduras, heridas quirúrgicas y trauma, } \\
\text { cirugía plástica }\end{array}$ \\
\hline \multicolumn{3}{|c|}{ 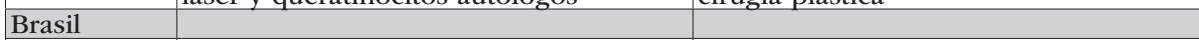 } \\
\hline Baumer & $\begin{array}{l}\text { GenOx Org }{ }^{\circledR} \\
\text { GenDerm } \AA \\
\text { GenOx Inorg® } \\
\text { GenMix } \AA \\
\text { OrthoGen } ® \\
\text { GenPhos }{ }^{\circledR} \text { HA TCP } \\
\end{array}$ & $\begin{array}{l}\text { Procedimientos de implantología buco } \\
\text { maxilofacial y cirugías óseas en general. }\end{array}$ \\
\hline Cellpraxis ${ }^{\circledR}$ & $\begin{array}{l}\text { ReACT® / Infusión de células madre me- } \\
\text { senquimales autólogas de médula ósea }\end{array}$ & Angina refractaria. \\
\hline \multicolumn{3}{|l|}{ Chile } \\
\hline Inbiocriotec & $\begin{array}{l}\text { SIOp/Membrana porosa biocompatible } \\
\text { y biodegradable de composición no es- } \\
\text { pecificada en el sitio web de la empresa } \\
\text { SIOc / Membrana porosa biocompatible } \\
\text { y biodegradable de composición no es- } \\
\text { pecificada en el sitio web de la empresa }\end{array}$ & $\begin{array}{l}\text { Heridas cutáneas (quemaduras, úlceras, } \\
\text { trauma, zonas donantes) } \\
\text { Tratamiento de lesiones de cartílago }\end{array}$ \\
\hline
\end{tabular}




\begin{tabular}{|c|c|c|}
\hline $\begin{array}{l}\text { Laboratorios } \\
\text { Recalcine }\end{array}$ & Biopiel@, membrana de quitosano & $\begin{array}{l}\text { Heridas cutáneas (quemaduras, ulceras, } \\
\text { trauma, zonas donantes) }\end{array}$ \\
\hline \multicolumn{3}{|l|}{ Colombia } \\
\hline $\begin{array}{l}\text { Inst. de Re- } \\
\text { generación } \\
\text { Tisular }\end{array}$ & Cytogel@, plasma rico en plaquetas & $\begin{array}{l}\text { Regeneración ósea, tratamiento de } \\
\text { lesiones articulares, úlceras en piel, } \\
\text { medicina estética. }\end{array}$ \\
\hline BioMaster ${ }^{\circledR}$ & $\begin{array}{l}\text { Bio M Colágeno/ Submucosa intestinal } \\
\text { porcina decelularizada } \\
\text { Bio M.3D/ Submucosa intestinal porcina } \\
\text { reforzada con titanio }\end{array}$ & $\begin{array}{l}\text { Heridas cutáneas (quemaduras, úlceras, } \\
\text { trauma, zonas donantes), urología y } \\
\text { neurología. } \\
\text { Aumento vertical de rebordes, rege- } \\
\text { neración ósea alrededor de mucosa, } \\
\text { implantes dentales, defectos periodon- } \\
\text { tales, preservación de alvéolos. }\end{array}$ \\
\hline 3Biomat & $\begin{array}{l}\text { Biomec®/ Submucosa intestinal porcina } \\
\text { decelularizada } \\
\text { Biomec Cx® Refuerzo de tejidos } \\
\text { blandos/ Submucosa intestinal porcina } \\
\text { decelularizada } \\
\text { Biomec Cx® Sustituto dural/ Submuco- } \\
\text { sa intestinal porcina decelularizada } \\
\text { Biomec Cx® Conducto nervioso / Sub- } \\
\text { mucosa intestinal porcina decelularizada } \\
\text { Biomec Cx® Peyronie/ Submucosa } \\
\text { intestinal porcina decelularizada }\end{array}$ & $\begin{array}{l}\text { Heridas cutáneas (quemaduras, ulceras, } \\
\text { trauma, zonas donantes) } \\
\text { Eventrorrafias y herniorrafias (inguinales, } \\
\text { umbilicales, hiatales y paraesofágicas). } \\
\text { Reemplazo de tejido dural en neurociru- } \\
\text { gía. } \\
\text { Regeneración de nervios periféricos. } \\
\text { Urología. }\end{array}$ \\
\hline $\begin{array}{l}\text { Keraderm } \\
\text { SAS }\end{array}$ & Keraderm $® /$ Queratinocitos autólogos & $\begin{array}{l}\text { Heridas cutáneas (quemaduras, ulceras, } \\
\text { trauma, zonas donantes). }\end{array}$ \\
\hline \multicolumn{3}{|l|}{ México } \\
\hline BioSkinCo & $\begin{array}{l}\text { Epifast }{ }^{\circledR} \text { Queratinocitos heterólogos } \\
\text { criopreservados sobre una gasa vaseli- } \\
\text { nada }\end{array}$ & $\begin{array}{l}\text { Heridas cutáneas (quemaduras, ulceras, } \\
\text { trauma, zonas donantes) }\end{array}$ \\
\hline
\end{tabular}

Tabla 18.3. Bancos de sangre de cordón umbilical en Latinoamérica

\begin{tabular}{|c|c|}
\hline Argentina & $\begin{array}{l}\text { BioCells, CrioCenter, Bioprocrearte, Protectia, Banco de Sangre del Cordón Umbi- } \\
\text { lical de Nueva Inglaterra, Matercell, Hospital Garrahan. }\end{array}$ \\
\hline Brasil & $\begin{array}{l}\text { Cord Vida, Cryopraxis, HemoMed, Banco de Sangre del Cordón Umbilical de } \\
\text { Nueva Inglaterra }\end{array}$ \\
\hline Chile & $\begin{array}{l}\text { Inco de Sangre del Cordón Umbilical de Nueva Inglaterra, Banco de Vida, Baby- } \\
\text { rd, Vida Cell, Inbiocriotec, Hemastem. }\end{array}$ \\
\hline Colombia & $\begin{array}{l}\text { Incelma, Instituto Antioqueño de Reproducción, STEM Medicina Regenerativa, } \\
\text { Cordón de Vida, RedCord, Hemocentro Distrital de la Secretaría de Salud de Bo- } \\
\text { gotá, Distrito Capital. }\end{array}$ \\
\hline Ecuador & Cryo-med, Biocells \\
\hline Méx & $\begin{array}{l}\text { DNA Vita Therapeutics, Banco de Cordón Umbilical, Mas Vision, Cryo-cell, Cordon } \\
\text { Vital, Biolife, Banco Central de Sangre, Banco de Células Madre del Hospital Uni- } \\
\text { versitario de la Universidad Autónoma de Nuevo León. }\end{array}$ \\
\hline Panamá & $\begin{array}{l}\text { Cryocell, Cordón de Vida, Banco de Sangre del Cordón Umbilical de Nueva Ingla- } \\
\text { terra. }\end{array}$ \\
\hline Perú & $\begin{array}{l}\text { Centro Peruano de Terapia Celular Regenerativa, Instituto de Criopreservación y } \\
\text { Terapia Celular, TERACELL Group, Banco de Cordón Umbilical del Perú, Células } \\
\text { Madre Brazzini, Lazo de Vida. }\end{array}$ \\
\hline Venezuela & $\begin{array}{l}\text { de Cordón Umbilical, Celulab, Banco de Sangre } \\
\text { nglaterra }\end{array}$ \\
\hline
\end{tabular}




\subsection{Algunos obstacúlos para la transferencia del laboratorio a la aplicación clínica}

Con base en nuestra experiencia y en la interacción con otros grupos de Colombia y Argentina que trabajan en ingeniería de tejidos y medicina regenerativa, así como, en las referencias bibliográficas encontradas [33, 34], en Latinoamérica los desafíos que enfrentan los científicos para desarrollar productos de aplicación clínica están relacionados con:

- Acceso limitado a recursos para investigación;

- Falta de formación académica de posgrado en el área;

- Dependencia de materiales importados;

- Falta de visibilidad de los grupos que trabajan en el área;

- Falta de redes de cooperación efectivas entre los grupos de investigación de cada país y de los diferentes países;

- Falta de alianzas entre las universidades y centros académicos con los hospitales y empresas.

Algunos de los grupos de investigación encontrados tienen filiación a instituciones que no se dedican a temas relacionados con la medicina regenerativa, como es el caso de los laboratorios de acelerador de electrones en Ecuador y de aprovechamiento sostenible de recursos naturales en México. Trasladar la investigación de estos grupos puede dificultarse, debido a que por el interés de la institución a la que están vinculados, no tienen comunicación directa con el desarrollo de productos ITMR y su aplicación clínica en el campo de la salud humana y veterinaria. De las limitaciones mencionadas, a continuación solo comentaremos las que consideramos más importantes.

\subsubsection{Acceso limitado a recursos para investigación}

La falta de recursos para investigación es resultado del bajo presupuesto que los gobiernos de la región destinan para este rubro. La Red 
de Indicadores de Ciencia y Tecnología Iberoamericana e Interamericana estableció que en el 2009 el promedio de la inversión en investigación y desarrollo experimental en los países latinoamericanos, fue de 52.5 dólares por habitante; la inversión más baja fue la de Guatemala con 1,49 y la más alta la de Brasil con 98.79. En este mismo periodo, Estados Unidos invirtió 1306 dólares por habitante, monto 25 veces mayor que el promedio en Latinoamérica. En la información publicada por la misma fuente, se encuentra que en Latinoamérica y el Caribe el aumento del Producto Interno Bruto (PIB) destinado a investigación fue solo del $0.06 \%$ en el periodo comprendido entre 1999 y el 2009; contrariamente, en los Estados Unidos el aumento fue de 0.2\% del PIB [87]. Aunque estos datos corresponden al total de los dineros invertidos en todos los campos de investigación, es de esperar que dada la baja divulgación de la naturaleza y bondades de los productos ITMR, la inversión en su investigación sea muy baja.

\subsubsection{Formación académica en ingeniería de tejidos y medicina regenerativa}

En Latinoamérica no hay programas formales de posgrado, maestría y doctorado, de formación en ingeniería de tejidos y medicina regenerativa. Existen posgrados en materiales, ingeniería biomédica, bioingeniería, ciencias farmacéuticas, odontología, biotecnología, ciencias biomédicas, etc., que alimentan con sus profesores y estudiantes a los grupos de investigación en esta área. Por ejemplo, en Colombia, los investigadores y estudiantes de posgrado se han ido formando en el transcurso de la ejecución de sus tesis y a través de la participación en cursos locales o internacionales, como: “Advances on Tissue Engineering” Rice University, Houston, Texas, USA; Primer Curso Congreso Internacional en Ingeniería de Tejidos y Medicina Regenerativa, Universidad Nacional de Colombia, Bogotá, Colombia (Avalado por la Sociedad Internacional de Ingeniería de Tejidos y Medicina Regenerativa-TERMIS); Segundo Curso Congreso Internacional en Ingeniería de Tejidos y Medicina Regenerativa, 
Universidad de Antioquia, Medellín, Colombia; Curso Internacional de Ingeniería de Tejidos y Medicina Regenerativa, Universidad Peruana Cayetano Heredia, Lima, Perú [4, 88-90].

En la región existe una sociedad científica denominada "Sociedad Latinoamericana de Biomateriales, Ingeniería de Tejidos y Órganos Artificiales" (SLABO), que se originó y tiene su sede en Brasil. Cada dos años, lleva a cabo talleres internacionales en los que participan profesores y estudiantes de pre y posgrado especialmente de países del cono sur (Chile, Argentina, Brasil, Uruguay, Paraguay). Los dos últimos talleres fueron organizados en el 2009 y en el 2011, en Argentina, con la Facultad de Ciencias Bioquímicas y Farmacéuticas de la Universidad de Rosario y con la Facultad de Ingeniería de la Universidad Nacional del Mar del Plata, respectivamente [91]. La Sociedad Internacional de Ingeniería de Tejidos y Medicina Regenerativa (TERMIS), que agrupa internacionalmente a la comunidad científica del área, cuenta con un capítulo de las Américas de reciente creación, en el que participan investigadores de varios países latinoamericanos. Éste inició cuando el capítulo de Norteamérica cambio su denominación para incluir a investigadores de Centro y Suramérica, con el fin de fortalecer la comunicación y el intercambio académico en el continente [92].

\subsubsection{Manufactura local de productos ITMR}

En general los productos de ingeniería de tejidos dependiendo de su complejidad, requieren tiempos de producción prolongados, son costosos y deben ser aplicados por profesionales de la salud con experiencia en su manejo para evitar fallas terapéuticas. Su producción enfrenta limitaciones impuestas por su composición y naturaleza y por la carencia de modelos de negocio que exitosamente permitan el paso del laboratorio a la clínica. Estos factores han contribuido a que en el mundo su implementación clínica se haya llevado a cabo con lentitud y a que su popularización haya sido limitada $[93,94]$. Considerando la baja inversión estatal y de la empresa privada que en Latinoamérica se hace en investigación 
y desarrollo en el campo, es posible que el posicionamiento de estos productos como alternativas terapéuticas reales lleve más tiempo. Lo descrito, aunado al hecho de que el grueso de los profesionales de la salud que los deben aplicar desconocen la mayoría de los tratamientos con productos ITMR y la forma de aplicación de los mismos, muestra la importancia de hacer una divulgación extensiva de las ventajas que estas terapias tienen sobre las terapias convencionales.

Como se muestra en las Tablas 18.2 y 18.3, en Latinoamérica hay producción y comercialización de soportes, terapias celulares y servicios de criopreservación de sangre de cordón umbilical. Los soportes producidos localmente son distribuidos como dispositivos médicos y al no contener células, pueden llegar a ser los que más rápido se comercialicen porque no son tan complejos y ya han sido introducidos por compañías farmacéuticas multinacionales [95], esto a pesar de ser su competencia en el mercado. Nuestro grupo de investigación (Grupo de trabajo en ingeniería de tejidos de la Universidad Nacional de Colombia), ha incursionado en el desarrollo de soportes tridimensionales de colágeno I y su evaluación pre-clínica en modelos de herida de mucosa oral $[16,55]$ y clínica, en pacientes con úlceras venosas, diabéticas y por presión. En su implementación clínica, se han encontrado obstáculos relacionados con la falta de relación entre los científicos que llevan a cabo los desarrollos y el personal de salud que debe prescribirlos, demoras en el proceso de patente y en el acondicionamiento de la logística requerida para su escalamiento a nivel industrial y aprobación por parte de la entidad regulatoria nacional. A pesar de nuestra participación en procesos de emprendimiento, la transferencia de este producto a la clínica se ha retrasado por la falta de mecanismos que faciliten la creación de alianzas Universidad- Empresa y la creación de empresas spin-off en la Universidad Nacional de Colombia, sitio en el que se adelantaron las investigaciones que condujeron a este desarrollo.

La implementación clínica de los tejidos y órganos artificiales puede ser más difícil, debido a su viabilidad y a que la tendencia es que sean elaborados con células provenientes de cultivos obtenidos a partir de muestras del mismo paciente [93, 94]. El empleo de tejido artificial autólogo requiere tomar y procesar tejido para aislar y cultivar las células 
de interés; también, sembrar las células en soportes y el cultivo de estos hasta que el tejido artificial esté listo para ser injertado en el paciente. Por consiguiente, en su producción y uso es necesario contar con instalaciones que brinden condiciones de esterilidad para tomar biopsias, aislar las células, cultivar el tejido, empacar y finalmente garantizar su entrega para el procedimiento quirúrgico. Lo anterior, implica la existencia de una infraestructura de producción adecuada y de una red de clínicos entrenados en el manejo y aplicación del tejido artificial; además, la existencia de una logística que garantice su transporte adecuado y aplicación en el margen de tiempo determinado por la viabilidad del producto (generalmente pocos días).

\subsection{Conclusiones}

En Latinoamérica los soportes tridimensionales bioactivos acelulares ( $\sin$ células), son un insumo importante de los tratamientos adelantados por las clínicas de heridas, médicos, odontólogos y veterinarios, para mejorar la curación de lesiones de piel, mucosa y hueso. Como ya mencionamos, algunos países del área los producen, sin embargo, la mayoría siguen siendo elaborados e importados por multinacionales como Johnson \& Johnson, Biomet 3I INC, Tecnoss, Biomantle, BaumerGenius, Zimmer Dental, Biomantle, Audio Technologies, etc. En Colombia, el acceso de la mayoría de la población a estos productos está limitado por los precios de venta y por el hecho de que el sistema de seguridad social no los cubre [96]. Por eso, es importante ampliar su producción local con tecnologías que aseguren la calidad y disminuyan su costo.

En la región latinoamericana los sustitutos vivos de piel constituyen una buena oportunidad de promover la aplicación de los productos ITMR, debido a que no son fáciles de conseguir e importar. En 1998 Apligraf®, piel artificial elaborada con soportes de colágeno tipo I sembrados con células de prepucio de neonato (fibroblastos y queratinocitos), fue aprobado por la FDA para el tratamiento de úlceras crónicas en pacientes diabéticos o con problemas vasculares; sin embargo, solo puede ser 
adquirida y aplicada por médicos entrenados por Organogenesis, la compañía que la elabora [3]. Adicionalmente, por tratarse de un tejido vivo hay dificultades asociadas con su costo, transporte e importación que dificultan su adquisición y aplicación. Otra oportunidad de implementar clínicamente las terapias ITMR, son los sustitutos de cartílago elaborados cultivando condrocitos autólogos en soportes de colágeno tipo II. Debido a que requieren que las células sean obtenidas a partir de biopsias del cartílago articular de la rodilla del paciente, solo pueden ser aplicados en donde existe la logística requerida para su producción. Un ejemplo de estos productos es BioCart ${ }^{\mathrm{TM}}$, desarrollado por una compañía Israelí el cual solo se vende en este país y en Italia en donde la compañía productora avala una clínica para su aplicación [97]. En los casos descritos, los pacientes latinoamericanos que pueden y quieren acceder a estos tejidos artificiales deben desplazarse a las clínicas autorizadas para aplicar el procedimiento, lo cual aumenta considerablemente el costo.

La implementación de tratamientos basados en el injerto de tejidos y órganos artificiales en Latinoamérica, no ha superado la barrera de los ensayos clínicos, algunos de los cuales evalúan productos desarrollados por centros de investigación consolidados de países desarrollados. En este marco, un grupo latinoamericano tuvo la iniciativa de formar una red con el Instituto McGowan para Medicina Regenerativa de la Escuela Médica de la Universidad de Pittsburgh y el Instituto Wake Forest para Medicina Regenerativa de la Universidad de Wake Forest, de los Estados Unidos. Dentro de esta colaboración se desarrolló en el Hospital Universitario Austral de Buenos Aires el concepto de la "Unidad de Traslación Clínica" (CTU-Clinical Translation Unit). Las instalaciones de la CTU integraban completamente un cuarto de cultivo celular con la sala de cirugía, evitando que las células abandonaran la sala de cirugía [98]. Creemos que el modelo de la unidad de traslación clínica muestra uno de los posibles caminos para la implementación clínica de productos de ingeniería de tejidos en Latinoamérica. Sin embargo, pocos años después de ser inaugurada fue cerrada sin alcanzar sus proyecciones, al resultar económicamente inviable debido a la poca conexión entre la academia y las empresas privadas y al poco interés de estas últimas en invertir en investigación y desarrollo en los países emergentes (comuni- 
cación personal con el Dr. Alejandro Nieponice, ex-director).Para que en los países latinoamericanos se pueda dar el crecimiento de la ingeniería de tejidos y la medicina regenerativa además de desarrollar investigación, se necesita formar personal con la experiencia que soporte el proceso de desarrollo y aplicación de tejido artificial autólogo; así como, contar con una infraestructura especializada. También, es indispensable llevar a cabo una labor extensiva de difusión y educación orientada a lograr que los gobiernos, los sistemas de salud y el personal clínico que lo conforma entiendan las ventajas de implementar clínicamente estas terapias. Una vez se haya logrado este convencimiento, será más fácil lograr que los pacientes acepten la aplicación de productos ITMR.

\subsection{Agradecimientos}

A la Universidad Nacional de Colombia, por estar dando los primeros pasos hacia la creación de una empresa spin-off basada en productos ITMR, desarrollados por el Grupo de Trabajo en Ingeniería de Tejidos de la Universidad Nacional de Colombia. A RIMADEL, por crear el espacio de colaboración que hizo posible la existencia de este capítulo. A Colciencias-SENA y a la División de Investigaciones de la Sede Bogotá de la Universidad Nacional de Colombia (DIB) por financiar la investigación que hemos adelantado y a Edward Suesca (Proyecto 10287 Colciencias y Proyecto 7795 DIB) y Sergio Casadiegos (Proyectos 9339 y 14704 DIB).

\subsection{Referencias}

[1] R. Langer, J.P. Vacanti, Science. 1993, 260, 920-6.

[2] E. Lavik, R. Langer, Appl Microbiol Biotechnol. 2004, 65, 1-8.

[3] L. Zaulyanov, R.S. Kirsner, Clin Interv Aging. 2007, 2, 93-8.

[4] C.S. Hankin, J. Knispel, M. Lopes, A. Bronstone, E. Maus, J Manag Care Pharm. 2012, 18, 375-84.

[5] A. Atala, J Endourol. 2000, 14, 49-57.

[6] A. Atala, Curr Stem Cell Res Ther. 2008, 3, 21-31. 
[7] C. De Bie, Regen Med. 2007, 2, 95-7.

[8] K.H. Lee, Yonsei Med J. 2000, 41, 774-9.

[9] L. Chan, T. Daim, Futures. 2012, 44 618-630.

[10] I.V. Yannas, Biomaterials. 2013, 34, 321-30.

[11] L.A. Fortier, Vet Surg. 2005, 34, 415-23.

[12] J.M. Moraleda, M. Blanquer, P. Bleda, P. Iniesta, F. Ruiz, S. Bonilla, C. Cabanes, L. Tabares, S. Martinez, Transpl Immunol. 2006, 17, 74-7.

[13] A. Atala, J Tissue Eng Regen Med. 2007, 1, 83-96.

[14] S.V. Murphy, A. Atala, Bioessays. 2012.

[15] M.B. Fisher, R.L. Mauck, Tissue Eng Part B Rev. 2013, 19, 1-13.

[16] M.R. Fontanilla, L.G. Espinosa, Tissue Eng Part A. 2012, 18, 1857-66.

[17] D.P. Bottaro, A. Liebmann-Vinson, M.A. Heidaran, Ann N Y Acad Sci. 2002, 961, 143-53.

[18] Website: Instituto Nacional de Rehabilitación (INR), http://www.inr.gob.mx/i20.htm, (accessed June 20, 2014).

[19] Website: FDA, U.S. Food and Drug Administration, USA, http://www.fda.gov/ BiologicsBloodVaccines/GuidanceComplianceRegulatoryInformation/ComplianceActivities/ Enforcement/CompliancePrograms/ucm095207.htm\#a, (accessed June 20, 2014).

[20] Website: REGULATION (EC) No 1394/2007 OF THE EUROPEAN PARLIAMENT AND OF THE COUNCIL of 13 November 2007 on advanced therapy medicinal products and amending Directive 2001/83/EC and Regulation (EC) No 726/2004, http://eur-lex.europa.eu/LexUriServ/ LexUriServ.do?uri=OJ:L:2007:324:0121:0137:en:PDF, (accessed June 20, 2014).

[21] K.J. Burg, S. Porter, J.F. Kellam, Biomaterials. 2000, 21, 2347-59.

[22] Website: International Medical Device Regulators Forum (IMDRF), , http://www.imdrf. org/docs/ghtf/final/sg1/technical-docs/ghtf-sg1-n77-2012-principles-medical-devicesclassification-121102.pdf, (accessed June 20, 2014).

[23] K. Hofman, A. Primack, G. Keusch, S. Hrynkow, Am J Public Health. 2005, 95, 13-7.

[24] I.P. Chudi, Medical Practice and Reviews. 2010, 1, 9-11.

[25] A. Boutayeb, S. Boutayeb, Int J Equity Health. 2005, 4, 2.

[26] S.J. Marshall, Bull World Health Organ. 2004, 82, 556.

[27] C. Giannou, M. Baldan, (Eds.), Cirugía de Guerra Trabajar con Recursos Limitados en Conflictos Armados y Otras Situaciones de Violencia. capitulos 3,4,5, 2011.

[28] Y. Sawa, S. Miyagawa, T. Sakaguchi, T. Fujita, A. Matsuyama, A. Saito, T. Shimizu, T. Okano, Surg Today. 2012, 42, 181-4.

[29] M.J. Elliott, P. De Coppi, S. Speggiorin, D. Roebuck, C.R. Butler, E. Samuel, C. Crowley, C. McLaren, A. Fierens, D. Vondrys, L. Cochrane, C. Jephson, S. Janes, N.J. Beaumont, T. Cogan, A. Bader, A.M. Seifalian, J.J. Hsuan, M.W. Lowdell, M.A. Birchall, Lancet. 2012, 380, 994-1000.

[30] P. Fagerholm, N.S. Lagali, D.J. Carlsson, K. Merrett, M. Griffith, Clin Transl Sci. 2009, $2,162-4$.

[31] S. Cebotari, A. Lichtenberg, I. Tudorache, A. Hilfiker, H. Mertsching, R. Leyh, T. Breymann, K. Kallenbach, L. Maniuc, A. Batrinac, O. Repin, O. Maliga, A. Ciubotaru, A. Haverich, Circulation. 2006, 114, I132-7.

[32] M. Nagata, H. Hoshina, M. Li, M. Arasawa, K. Uematsu, S. Ogawa, K. Yamada, T. Kawase, K. Suzuki, A. Ogose, I. Fuse, K. Okuda, K. Uoshima, K. Nakata, H. Yoshie, R. Takagi, Bone. 2012, 50, 1123-9. 
[33] H.L. Greenwood, P.A. Singer, G.P. Downey, D.K. Martin, H. Thorsteinsdottir, A.S. Daar, PLoS Med. 2006, 3, e381.

[34] H.L. Greenwood, H. Thorsteinsdóttir, G. Perry, James Renihan, P.A. Singer, A.S. Daar, Int. J. Biotechnology. 2006, 8, 60-77.

[35] Website: Centro de Investigación en Ingeniería de Tejidos y Terapias Celulares (CIITTC). Universidad Maimónides, http://www.maimonides.edu.ar/es/invesCIITT.php, (accessed June 20, 2014).

[36] G.A. Moviglia, N. Blasetti, J.O. Zarate, D.E. Pelayes, Ophthalmic Research. 2012, 48, 1-5.

[37] Website: Centro Único Coordinador de Ablación e Implante, Buenos Aires, http://www. cucaiba.gba.gov.ar/ingenieria.htm, (accessed June 20, 2014).

[38] E. Mansilla, R. Spretz, G. Larsen, L. Nunez, H. Drago, F. Sturla, G.H. Marin, G. Roque, K. Martire, V. Diaz Aquino, S. Bossi, C. Gardiner, R. Lamonega, N. Lauzada, J. Cordone, J.C. Raimondi, J.M. Tau, N.R. Biasi, J.E. Marini, A.N. Patel, T.E. Ichim, N. Riordan, A. Maceira, Transplant Proc. 2010, 42, 4275-8.

[39] E. Mansilla, K. Mártire, G. Roque, J.M. Tau, G.H. Marín, M.V. Gastuma, G. Orlandi, A. Tarditti, Journal of Transplant Technolies \& Research. 20|3, 3.

[40] P.C. Caracciolo, V. Thomas, Y.K. Vohra, F. Buffa, G.A. Abraham, J Mater Sci Mater Med. 2009, 20, 2129-37.

[41] Biomateriais para Implante em tecido ósseo. http://www.forp.usp.br/pesquisa/index. php?option $=$ com_content\&view $=$ article $\& i d=157$ :biomateriais-para-implante-em-tecidoosseo\&catid=22: depcirurgiatraumat-buco-maxilo-facial-e-period\&Itemid=9: $\quad$ accessed

[42] S. Saska, R.M. Scarel-Caminaga, L.N. Teixeira, L.P. Franchi, R.A. Dos Santos, A.M. Gaspar, P.T. de Oliveira, A.L. Rosa, C.S. Takahashi, Y. Messaddeq, S.J. Ribeiro, R. Marchetto, J Mater Sci Mater Med. 2012, 23, 2253-66.

[43] REPARO E BIOCOMPATIBILIDADE. http://www.forp.usp.br/pesquisa/index.php? option=com_content $\& v i e w=$ article $\&$ id=152:reparo-e-biocompatibilidade $\&$ catid=17:depmorfologia-estomatologia-e-fisiologia\&Itemid=9: (accessed

[44] S.A. Lacerda, J.F. Lanzoni, K.F. Bombonato-Prado, A.A. Campos, C.A. Prata, L.G. Brentegani, Implant Dent. 2009, 18, 521-9.

[45] S.M. Friedman, C.A. Gamba, P.M. Boyer, M.B. Guglielmotti, M.I. Vacas, P.N. Rodriguez, C. Guerrero, F. Lifshitz, International Journal of Food Sciences and Nutrition. 2001, 52, 225-233.

[46] M.A. Batista, T.P. Leivas, C.J. Rodrigues, G.C. Arenas, D.R. Belitardo, R. Guarniero, Clinics (Sao Paulo). 2011, 66, 1787-92.

[47] Website: Heart Institute (InCor). Universidade de São Paulo. Escuela de medicina., http://www.incor.usp.br/sites/webincor.15/, (accessed June 20, 2014).

[48] A. Kaasi, I.A. Cestari, N.A. Stolf, A.A. Leirner, O. Hassager, I.N. Cestari, J Tissue Eng Regen Med. 2011, 5, 292-300.

[49] Website: Centro de Investigación en Criogenia e Ingeniería en Tejidos (CICRIT), http:// sitios.upla.cl/contenidos/2009/06/30/se-inauguro-1er-centro-en-criogenia-e-ingenieriade-tejidos-de-chile/, (accessed June 20, 2014).

[50] C.R. Weinstein-Oppenheimer, A.R. Aceituno, D.I. Brown, C. Acevedo, R. Ceriani, M.A. Fuentes, F. Albornoz, C.F. Henriquez-Roldan, P. Morales, C. Maclean, S.M. Tapia, M.E. Young, J Transl Med. 2010, 8, 59.

[51] C.A. Acevedo, R.A. Somoza, C. Weinstein-Oppenheimer, S. Silva, M. Moreno, E. Sanchez, F. Albornoz, M.E. Young, W. Macnaughtan, J. Enrione, Bioprocess Biosyst Eng. 2013, 36, 317-24. 
[52] C.A. Acevedo, R.A. Somoza, C. Weinstein-Oppenheimer, D.I. Brown, M.E. Young, Biotechnol Lett. 2010, 32, 1011-7.

[53] A. Vidal, A. Giacaman, F.A. Oyarzun-Ampuero, S. Orellana, I. Aburto, M.F. Pavicic, A. Sanchez, C. Lopez, C. Morales, M. Caro, I. Moreno-Villoslada, M. Concha, Am J Ther. 2013, 20, 394-8.

[54] Website: Grupo de Trabajo en Ingeniería de Tejidos. Universidad Nacional de Colombia, http://www.tringtejidos.unal.edu.co/index.html, (accessed June 20, 2014).

[55] L. Espinosa, A. Sosnik, M.R. Fontanilla, Tissue Eng Part A. 2010, 16, 1667-79.

[56] Website: Universidad de Los Andes. Grupo de Ingenieria Biomedica. , https:// ingbiomedica.uniandes.edu.co/, (accessed June 20, 2014).

[57] T. Gardeazabal, M. Cabrera, P. Cabrales, M. Intaglietta, J.C. Briceno, J Appl Physiol. 2008, 105, 588-94.

[58] Website: Universidad De Antioquia - Grupo Ingeniería de tejidos y terapias celulares. , http://201.234.78.173:8080/gruplac/jsp/visualiza/visualizagr.jsp?nro=00000000000840, (accessed June 20).

[59] M. Arango, C. Chamorro, O. Cohen-Haguenauer, M. Rojas, L.M. Restrepo, Dermatol Online J. 2005, 11, 2.

[60] Grupo de Investigación en Biomateriales. BIOMAT. http://jaibana.udea.edu.co/programas/ bioingenieria/index/index.php?page=grupos_investigacion: (accessed

[61] Website: Universidad de Caldas http://201.234.78.173:8080/gruplac/jsp/visualiza/ visualizagr.jsp?nro=00000000006822, (accessed June 20, 2014).

[62] J.U. Carmona, C. Lopez, Journal of Equine Veterinary Science. 2011, 31, 506-510.

[63] N. Higuita-Castro, D. Gallego-Perez, A. Pelaez-Vargas, F. Garcia Quiroz, O.M. Posada, L.E. Lopez, C.A. Sarassa, P. Agudelo-Florez, F.J. Monteiro, A.S. Litsky, D.J. Hansford, J Biomed Mater Res B Appl Biomater. 2011.

[64] Website: Instituto Tecnológico de Costa Rica. Centro de Investigación en Biotecnolgía (CIB), http://www.tec.ac.cr/sitios/Docencia/biologia/cib/Paginas/default.aspx, (accessed June 20, 2014).

[65] H. Peniche, F. Reyes-Ortega, M.R. Aguilar, G. Rodriguez, C. Abradelo, L. Garcia-Fernandez, C. Peniche, J.S. Roman, Macromol Biosci. 2013.

[66] J. Berlanga, J.I. Fernandez, E. Lopez, P.A. Lopez, A. del Rio, C. Valenzuela, J. Baldomero, V. Muzio, M. Raices, R. Silva, B.E. Acevedo, L. Herrera, MEDICC Rev. 2013, 15, 11-5.

[67] T. Ramirez, Revista Politecnica. 2008, 1, 138-142.

[68] A. Fonseca-Garcia, J.D. Mota-Morales, I.A. Quintero-Ortega, Z.Y. Garcia-Carvajal, V. Martinez-Lopez, E. Ruvalcaba, L. Solis, C. Ibarra, M.C. Gutierrez, M. Terrones, I.C. Sanchez, F. Del Monte, M.C. Velasquillo, G. Luna-Barcenas, J Biomed Mater Res A. 2013.

[69] D. Guzman-Uribe, K.N. Estrada, J. Guillen Ade, S.M. Perez, R.R. Ibanez, Open Dent J. 2012, 6, 226-34.

[70] Website: Centro de Investigación en Alimentación y Desarrollo, A.C. SubPrograma: Polímeros Naturales., http://www.ciad.mx/tecnologia-alimentos/polimeros-naturales. htm, (accessed June 20, 2014).

[71] M. Recillas, L.L. Silva, C. Peniche, F.M. Goycoolea, M. Rinaudo, W.M. Arguelles-Monal, Biomacromolecules. 2009, 10, 1633-41.

[72] Departamento de Biología Celular y Tisular. http://www.facmed.unam.mx/deptos/ biocetis/laboratorio\%20de\%20inmunoterapia\%20experimental.html: (accessed

[73] H. Pérez Campos, M. Saldias, G. Sanchez, P. Martucci, M. Acosta, R. Faccio, L. Suescun, M. Romero, A. Mombru, Cryobiology. 2012, 65, 340-341. 
[74] I.A. Chaim, M.A. Sabino, M. Mendt, A.J. Muller, D. Ajami, J Tissue Eng Regen Med. 2012, 6, 272-9.

[75] Website: Laboratorio de Bioingeniería de Tejidos. Universidad Simón Bolívar, https:// sites.google.com/site/bioingenieriadetejidosusb/, (accessed June 20, 2014).

[76] A.M. Ferreira, G. Gonzalez, R.J. Gonzalez-Paz, J.L. Feijoo, J. Lira-Olivares, K. NorisSuarez, Acta Microscopica. 2009, 18, 278-286.

[77] Biomaster. http://biomaster.co/producto_detalle.php?recordID=4: (accessed

[78] Website: FDA, U.S. Food and Drug Administration, USA, http://www.fda.gov/NewsEvents/ Newsroom/PressAnnouncements/2007/ucm108829.htm, (accessed June 20, 2014).

[79] P. Rubinstein, Bone Marrow Transplant. 2009, 44, 635-42.

[80] C. Gamba, M.A. Marcos, H. Trevani, J. Van der Velde, C.Y. Marcos, G. Theiler, J. Rossi, M. Zelasko, L. Fainboim, A.E. Del Pozo, Acta Bioquím Clín Latinoam. 2006, 40 491-497.

[81] Website: Banco de Cordón Umbilical BCU . http://www.bcu.com.mx/historia/, (accessed June 20, 2014).

[82] E.D. Calderón, Rev Med Inst Mex Seguro Soc. 2005, 43, 127-129.

[83] Website: Membracel, http://www.membracel.com.ar/, (accessed June 20, 2014).

[84] Website: Hyaltec, http://www.hyaltec.com.ar/, (accessed June 20, 2014 ).

[85] Website: Foundation for the Accreditation of Cellular Therapy., http://factwebsite.org/ CordSearch.aspx? \&type $=$ CordBloodBank\&country $=\&$ state $=,($ accessed June 20, 2014).

[86] Y.C. Hsu, E. Fuchs, Nat Rev Mol Cell Biol. 2012, 13, 103-14.

[87] La Red de Indicadores de Ciencia y Tecnología -Iberoamericana e Interamericana(RICYT): (accessed

[88] Website: Rice University. Department of Bioengineering, http://www.ruf.rice. edu/ mikosgrp/pages/ATE/ate.htm, (accessed June 20, 2014).

[89] J.E. Tengood, R. Ridenour, R. Brodsky, A.J. Russell, S.R. Little, Tissue Eng Part A. 2011, $17,1181-9$.

[90] Website: Universidad Peruana Cayetano Heredia. Escuela de Posgrado Victor Alzamora Castro., http://www.upch.edu.pe/epgvac/curso/62/curso-internacional-de-ingenieria-detejidos-y-medicina-regenerativa, (accessed June 20, 2014).

[91] Website: Sociedad Latinoamericana de Biomateriales, Ingeniería de Tejidos y Órganos Artificiales (SLABO), http://www.bioomat.fi.mdp.edu.ar/, (accessed June 20, 2014).

[92] S. Mirmalek Sani, Linking the international community of TERMIS. 2012, 7, 6.

[93] D.O. Fauza, Curr Opin Pediatr. 2003, 15, 267-71.

[94] D.J. Williams, I.M. Sebastine, IEE Proc Nanobiotechnol. 2005, 152, 207-10.

[95] R. Vernal, Revista Dental de Chile. 2001, 92., 33-44.

[96] Website: Ministerio de Salud y Protección Social, http://www.pos.gov.co/Paginas/ Medicamentos.aspx, (accessed June 20, 2014).

[97] Website: ProChon Biotech, Ltd., http://www.prochon.com/about-2/market-overview/, (accessed June 20, 2014).

[98] A. Nieponice, Clinical Translation of Tissue Engineering and Regenerative Medicine Technologies Alejandro Niepo, in Biomaterials and Stem Cells in Regenerative Medicine, C. Press, Editor. 2012. p. 521-532. 